\title{
Symmetries for Light-Front Quantization of Yukawa Model with Renormalization
}

Received: 12 January 2017 / Accepted: 24 August 2017 / Published online: 12 September 2017

(C) The Author(s) 2017. This article is an open access publication

\begin{abstract}
In this work we discuss the Yukawa model with the extra term of self-interacting scalar field in $D=1+3$ dimensions. We present the method of derivation the light-front commutators and anti-commutators from the Heisenberg equations induced by the kinematical generating operator of the translation $P^{+}$. Mentioned Heisenberg equations are the starting point for obtaining this algebra of the (anti-) commutators. Some discrepancies between existing and proposed method of quantization are revealed. The Lorentz and the CPT symmetry, together with some features of the quantum theory were applied to obtain the two-point Wightman function for the free fermions. Moreover, these Wightman functions were computed especially without referring to the Fock expansion. The Gaussian effective potential for the Yukawa model was found in the terms of the Wightman functions. It was regularized by the space-like point-splitting method. The coupling constants within the model were redefined. The optimum mass parameters remained regularization independent. Finally, the Gaussian effective potential was renormalized.
\end{abstract}

\section{From Heisenberg Equations to the Algebra of (Anti-) Commutators}

We suggest the approach for obtaining the algebra of the (anti-) commutators for the Yukawa model, which uses the Heisenberg equations induced by the kinematical operator of the translation $P^{+}$. These Heisenberg equations are treated as the jumping-off point for obtaining this algebra of the (anti-) commutators on the light front. This method allows to skip the classical canonical structure of theory, starting directly from the quantum level of the Heisenberg equations. It reveals some discrepancies between existing method and proposed herein the way of the quantization. This approach permits to derive the (anti)-commutators for the light-front canonical fields. To this end we study the Yukawa model with additional term of the self-interacting scalar field. This model is described in $D=1+3$ dimensions by the Lagrangian density

$$
\mathcal{L}=\frac{1}{2}(\partial \phi)^{2}-\frac{1}{2} m^{2} \phi^{2}-\lambda \phi^{4}+\bar{\Psi}(i \not \partial-M) \Psi-g \phi \bar{\Psi} \Psi,
$$

where $g$ denotes the coupling constant between fermionic fields $\Psi, \bar{\Psi}$ with the mass $M$ and the scalar field $\phi$ with the mass $m$. The coupling constant for self-interaction of the scalar field is $\lambda$. The explicit light-front formula of the above Lagrangian density satisfies

J. Żochowski $(\varangle) \cdot$ J. A. Przeszowski

Faculty of Physics, University of Białystok, Ul. Ciołkowskiego 1L, 15-245 Białystok, Poland

E-mail: j.zochowski@uwb.edu.pl

J. A. Przeszowski

E-mail: j.przeszowski@uwb.edu.pl 


$$
\begin{aligned}
\mathcal{L}= & \left(\partial_{-} \phi\right)\left(\partial_{+} \phi\right)-\frac{1}{2}\left(\partial_{j} \phi\right)\left(\partial_{j} \phi\right)-\frac{1}{2} m^{2} \phi^{2}-\lambda \phi^{4} \\
& +i \sqrt{2} \Psi_{+}^{\dagger} \partial_{+} \Psi_{+}+i \sqrt{2} \Psi_{-}^{\dagger} \partial_{-} \Psi_{-}+\frac{i}{\sqrt{2}} \Psi_{-}^{\dagger} \gamma^{+} \gamma^{j} \partial_{j} \Psi_{+}+\frac{i}{\sqrt{2}} \Psi_{+}^{\dagger} \gamma^{-} \gamma^{j} \partial_{j} \Psi_{-} \\
& -\frac{1}{\sqrt{2}} M \Psi_{-}^{\dagger} \gamma^{+} \Psi_{+}-\frac{1}{\sqrt{2}} M \Psi_{+}^{\dagger} \gamma^{-} \Psi_{-}-\frac{1}{\sqrt{2}} g \phi \Psi_{-}^{\dagger} \gamma^{+} \Psi_{+}-\frac{1}{\sqrt{2}} g \phi \Psi_{+}^{\dagger} \gamma^{-} \Psi_{-} .
\end{aligned}
$$

From the principle of minimal action we may derive the equations of motion for this model:

$$
\begin{aligned}
2 i \partial_{\mp} \Psi_{\mp}+i \gamma^{ \pm} \partial_{\perp} \Psi_{ \pm}-(M+g \phi) \gamma^{ \pm} \Psi_{ \pm} & =0, \\
\left(2 \partial_{-} \partial_{+}-\Delta_{\perp}\right) \phi+m^{2} \phi+4 \lambda \phi^{3}+\frac{1}{\sqrt{2}} g\left(\Psi_{-}^{\dagger} \gamma^{+} \Psi_{+}+\Psi_{+}^{\dagger} \gamma^{-} \Psi_{-}\right) & =0 .
\end{aligned}
$$

The Lagrangian density (2) leads to the following component of the canonical energy-momentum tensor

$$
T^{++}=\left(\partial_{-} \phi\right)^{2}+i \sqrt{2} \Psi_{+}^{\dagger} \partial_{-} \Psi_{+} .
$$

The component $T^{+-}$may be calculated in a similar way, using also the equations of motion for the Yukawa model (3). Therefore

$$
T^{+-}=\frac{1}{2}\left(\partial_{j} \phi\right)\left(\partial_{j} \phi\right)+\frac{1}{2} m^{2} \phi^{2}+\lambda \phi^{4}+i \sqrt{2} \Psi_{+}^{\dagger} \partial_{+} \Psi_{+} .
$$

Generators of the translations in the light-front directions $x^{\mu}$ are set by the integral

$$
P^{\mu}\left(x^{+}\right)=\int d^{3} \bar{y} T^{+\mu}\left(x^{+}, \bar{y}\right) .
$$

In the case of the kinematical operator $P_{-}=P^{+}[1,2]$, the Heisenberg equations obey:

$$
i \partial_{-} \Psi_{\mp}\left(x^{+}, \bar{x}\right)=\left[\Psi_{\mp}\left(x^{+}, \bar{x}\right), P_{-}\left(x^{+}\right)\right], \quad i \partial_{-} \phi\left(x^{+}, \bar{x}\right)=\left[\phi\left(x^{+}, \bar{x}\right), P_{-}\left(x^{+}\right)\right] .
$$

Each of these expressions is taken in the same, fixed parameter of the evolution $x^{+}$. They allow us to determine the canonical commutators and anti-commutators onto the hyper-surface of the constant light-front time $x^{+}$[3]. Thereby, the consequences of the relation (8) together with the translational invariance for the non-dynamical derivative $\partial_{-} \Psi_{-}$, expressed by the pattern (3), yield:

$$
\begin{aligned}
& \left\{\Psi_{-}\left(x^{+}, \bar{x}\right), \Psi_{+}^{\dagger}\left(x^{+}, \bar{y}\right)\right\}=-\frac{i}{2 \sqrt{2}}\left\{i \not \partial_{\perp}^{x}+\left[M+g \phi\left(x^{+}, \bar{y}\right)\right]\right\} \gamma^{+} \partial_{-}^{-x_{x}} \delta\left(x^{-}-y^{-}\right) \delta^{(2)}\left(\mathbf{x}_{\perp}-\mathbf{y}_{\perp}\right) \\
& \left\{\Psi_{-}\left(x^{+}, \bar{x}\right), \Psi_{+}\left(x^{+}, \bar{y}\right)\right\}=0, \quad\left[\phi\left(x^{+}, \bar{x}\right), \Psi_{-}\left(x^{+}, \bar{y}\right)\right]=0 .
\end{aligned}
$$

It is easy to show, that from the relation (8) we obtain the light-front (anti)-commutators:

$$
\begin{aligned}
\left\{\Psi_{+}\left(x^{+}, \bar{x}\right), \Psi_{+}^{\dagger}\left(x^{+}, \bar{y}\right)\right\} & =\frac{1}{\sqrt{2}} \delta^{(3)}(\bar{x}-\bar{y}) \Lambda_{+}, \\
\left\{\Psi_{+}\left(x^{+}, \bar{x}\right), \Psi_{+}\left(x^{+}, \bar{y}\right)\right\} & =0, \quad\left[\phi\left(x^{+}, \bar{x}\right), \Psi_{+}\left(x^{+}, \bar{y}\right)\right]=0 .
\end{aligned}
$$

The second of the Heisenberg Eq. (8) enables us to read out the canonical commutator for the scalar field

$$
\left[\phi\left(x^{+}, \bar{x}\right), \partial_{-}^{y} \phi\left(x^{+}, \bar{y}\right)\right]=\frac{i}{2} \delta^{(3)}(\bar{x}-\bar{y})
$$

and already inferred result (12). Remarkably, analogous Heisenberg equations with the generators $P_{+}$and $P_{j}$ do not lead to the new formulae of (anti)-commutators. In presented way: from the Heisenberg equations to the algebra of the (anti-) commutators, two of them $\left\{\Psi_{-}\left(x^{+}, \bar{x}\right), \Psi_{-}\left(x^{+}, \bar{y}\right)\right\}$ and $\left\{\Psi_{-}\left(x^{+}, \bar{x}\right), \Psi_{-}^{\dagger}\left(x^{+}, \bar{y}\right)\right\}$ remain undetermined. In contrast, the unmediated light-front calculation gives the following formula $\left\{\Psi_{-}\left(x^{+}, \bar{x}\right), \Psi_{-}\left(x^{+}, \bar{y}\right)\right\}=0$. As we shall perceive later, the anti-commutator $\left\{\Psi_{-}\left(x^{+}, \bar{x}\right), \Psi_{-}^{\dagger}\left(x^{+}, \bar{y}\right)\right\}$ is singular on the light-front hyper-surface in our approach. It differs from the existing result, which gives the studied anti-commutator as finite. Performed analysis reveals also, that in the Yukawa model one has $\Psi, \Psi^{\dagger}$ and $\phi$ as the light-front canonical quantum fields. 


\section{Integro-Differential Equations for Wightman Function}

Proposed method of the light-front quantization, together with some features of the quantum field theory, enables us to obtain the Wightman functions, either for the canonical fields or for those, which couldn't be treated in that way. The Euler-Lagrange equations of motion for the Yukawa model allow to write the relationships, binding together the components of the Wightman functions for fermions. In the second step of presented considerations they may be integrated, leading to the integro-(partial) differential equations. Such approach allows to proceed without the Fourier representation in the Fock expansion of the fermionic field, expressed in the terms of the creation and annihilation operators [4,5]. In this method the role of the Lorentz and the CPT symmetries is especially accentuated.

Components of the Wightman function for the non-interacting fermions arise from the Dirac equations of motion (3). For the decoupled fields $(g=0)$, we have:

$$
2 i \partial_{ \pm} S_{ \pm \pm}(x)=\left(i \not \partial_{\perp}+M\right) \gamma^{\mp} S_{\mp \pm}(x), \quad 2 i \partial_{ \pm} S_{ \pm \mp}(x)=\left(i \not \partial_{\perp}+M\right) \gamma^{\mp} S_{\mp \mp}(x) .
$$

First of these patterns can be integrated over the $x^{+}$variable

$$
S_{++}\left(x^{+}, x^{-}, \mathbf{x}_{\perp}\right)=S_{++}\left(0, x^{-}, \mathbf{x}_{\perp}\right)+\frac{i}{2} \gamma^{-}\left(i \not \partial_{\perp}-M\right) \int_{0}^{x^{+}} d \tau S_{-+}\left(\tau, x^{-}, \mathbf{x}_{\perp}\right) .
$$

The light-front hyper-surface term $S_{++}\left(0, x^{-}, \mathbf{x}_{\perp}\right)$ comes from the anti-commutator (11). Using the result (88) from the CPT theorem and the distribution identity $1 /(x \pm i \varepsilon)=\mathrm{P}(1 / x) \mp i \pi \delta(x)$, we find

$$
S_{++}\left(0, x^{-}, \mathbf{x}_{\perp}\right)=-\frac{i}{2 \sqrt{2} \pi} \cdot \frac{1}{x^{-}-i \varepsilon} \delta^{(2)}\left(\mathbf{x}_{\perp}\right) \Lambda_{+} .
$$

Inserting complete result for the $S_{++}\left(x^{+}, x^{-}, \mathbf{x}_{\perp}\right)$ into the (14), one has the integro-(partial) differential equation

$$
\begin{aligned}
\partial_{-} S_{-+}\left(x^{+}, x^{-}, \mathbf{x}_{\perp}\right)= & \frac{1}{4 \sqrt{2} \pi} \cdot \frac{1}{x^{-}-i \varepsilon} \gamma^{+}\left(i \not \partial_{\perp}-M\right) \delta^{(2)}\left(\mathbf{x}_{\perp}\right) \\
& +\frac{1}{2}\left(\Delta_{\perp}-M^{2}\right) \int_{0}^{x^{+}} d \tau S_{-+}\left(\tau, x^{-}, \mathbf{x}_{\perp}\right) .
\end{aligned}
$$

But taking account of the relativistic symmetry (81) of the Wightman function we may also obtain the derivative over light-front time $x^{+}$for the $S_{-+}\left(x^{+}, x^{-}, \mathbf{x}_{\perp}\right)$. After simple manipulations with distributions

$$
\begin{aligned}
\partial_{+} S_{-+}\left(x^{+}, x^{-}, \mathbf{x}_{\perp}\right)= & \frac{1}{4 \sqrt{2} \pi} \cdot \frac{1}{x^{+}-i \varepsilon} \gamma^{+}\left(i \not \partial_{\perp}-M\right) \delta^{(2)}\left(\mathbf{x}_{\perp}\right) \\
& +\frac{1}{2}\left(\Delta_{\perp}-M^{2}\right) \int_{0}^{x^{-}} d \xi S_{-+}\left(x^{+}, \xi, \mathbf{x}_{\perp}\right) .
\end{aligned}
$$

The function $S_{--}\left(x^{+}, x^{-}, \mathbf{x}_{\perp}\right)$ can be reproduced from the $S_{+-}\left(x^{+}, x^{-}, \mathbf{x}_{\perp}\right)$ by integration of (14). Finally one has the integral equation

$$
S_{--}\left(x^{+}, x^{-}, \mathbf{x}_{\perp}\right)=-\frac{i}{2 \sqrt{2} \pi} \cdot \frac{1}{x^{+}-i \varepsilon} \delta^{(2)}\left(\mathbf{x}_{\perp}\right) \Lambda_{-}+\frac{i}{2} \gamma^{+}\left(i \not \partial_{\perp}-M\right) \int_{0}^{x^{-}} d \xi S_{+-}\left(x^{+}, \xi, \mathbf{x}_{\perp}\right) .
$$

By studying the bi-linear invariants for the projection structure of the Wightman function for fermions, one can put following decomposition

$$
S(x)=\left\langle 0\left|\Psi(x) \Psi^{\dagger}(0)\right| 0\right\rangle=\not \partial \gamma^{0} B(x)+\gamma^{0} A(x) .
$$

These $A(x)$ and $B(x)$ are the two scalar functions. After the projection onto the $\gamma^{0}$ matrix, the expression (20) gives $\partial_{\mu} A(x)+i M \partial_{\mu} B(x)=0$ and $\partial^{2} B(x)+i M A(x)=0$, where also the Dirac equation $(i \not \gamma-M) \Psi(x)=0$ was applied. The consequences of the above relationships are the Klein-Gordon equations for the $A(x)$ and $B(x)$ functions: $\partial^{2} A(x)+M^{2} A(x)=0, \partial^{2} B(x)+M^{2} B(x)=0$. For the same reason, we have: 
$A(x)+i M B(x)=0, \partial^{2} A(x)-i M^{3} B(x)=0$. From the introduced decomposition (20) and by its projection onto the + and - components for the fermion field operator, we can establish:

$$
\begin{aligned}
& S_{ \pm \pm}(x)=\left\langle 0\left|\Psi_{ \pm}(x) \Psi_{ \pm}^{\dagger}(0)\right| 0\right\rangle=\sqrt{2} \partial_{\mp} B(x) \Lambda_{ \pm}, \\
& S_{ \pm \mp}(x)=\left\langle 0\left|\Psi_{ \pm}(x) \Psi_{\mp}^{\dagger}(0)\right| 0\right\rangle=-\frac{1}{\sqrt{2}} \gamma^{\mp} \partial_{\perp} B(x)+\frac{1}{\sqrt{2}} \gamma^{\mp} A(x) .
\end{aligned}
$$

It is easy to substitute the decomposition (21) into the Eqs. (15), (19) and to perform the projection by taking the trace. As the result one has the two integro-(partial) differential equations for the $B(x)$ function:

$$
\begin{aligned}
& \partial_{-} B\left(x^{+}, x^{-}, \mathbf{x}_{\perp}\right)=-\frac{i}{4 \pi} \cdot \frac{1}{x^{-}-i \varepsilon} \delta^{(2)}\left(\mathbf{x}_{\perp}\right)+\frac{1}{2}\left(\Delta_{\perp}-M^{2}\right) \int_{0}^{x^{+}} d \tau B\left(\tau, x^{-}, \mathbf{x}_{\perp}\right), \\
& \partial_{+} B\left(x^{+}, x^{-}, \mathbf{x}_{\perp}\right)=-\frac{i}{4 \pi} \cdot \frac{1}{x^{+}-i \varepsilon} \delta^{(2)}\left(\mathbf{x}_{\perp}\right)+\frac{1}{2}\left(\Delta_{\perp}-M^{2}\right) \int_{0}^{x^{-}} d \xi B\left(x^{+}, \xi, \mathbf{x}_{\perp}\right) .
\end{aligned}
$$

Doing the same operations, we can project the integro-differential Eqs. (17) and (18) onto the $\gamma^{\mp}$ matrices, respectively. Applying also the pattern (22) and taking the trace we derive the integro-(partial) differential equations for the $A(x)$ function:

$$
\begin{aligned}
& \partial_{-} A\left(x^{+}, x^{-}, \mathbf{x}_{\perp}\right)=-\frac{1}{4 \pi} \cdot \frac{M}{x^{-}-i \varepsilon} \delta^{(2)}\left(\mathbf{x}_{\perp}\right)+\frac{1}{2}\left(\Delta_{\perp}-M^{2}\right) \int_{0}^{x^{+}} d \tau A\left(\tau, x^{-}, \mathbf{x}_{\perp}\right), \\
& \partial_{+} A\left(x^{+}, x^{-}, \mathbf{x}_{\perp}\right)=-\frac{1}{4 \pi} \cdot \frac{M}{x^{+}-i \varepsilon} \delta^{(2)}\left(\mathbf{x}_{\perp}\right)+\frac{1}{2}\left(\Delta_{\perp}-M^{2}\right) \int_{0}^{x^{-}} d \xi A\left(x^{+}, \xi, \mathbf{x}_{\perp}\right) .
\end{aligned}
$$

The Wightman function for fermions obeys the condition of (80), being the corollary from the Lorentz symmetry. By regarding the decomposition (20) and using above method of the projection, the below result may be showed directly

$$
\left(x^{+} \partial_{j}+x^{j} \partial_{-}\right) A(x)=0 .
$$

But the projection onto the single matrix $\gamma^{k}, \gamma^{-}$or $\gamma^{+}$leads us respectively to:

$$
\begin{gathered}
\left(x^{+} \partial_{j}+x^{j} \partial_{-}\right) \partial_{k} B(x)+\delta_{j k} \partial_{-} B(x)=0, \quad\left(x^{+} \partial_{j}+x^{j} \partial_{-}\right) \partial_{+} B(x)+\partial_{j} B(x)=0, \\
\left(x^{+} \partial_{j}+x^{j} \partial_{-}\right) \partial_{-} B(x)=0 .
\end{gathered}
$$

The conditions of the Lorentz symmetry for the functions $A(x)$ and $B(x)$ may be solved onto the light-front hyper-surface $x^{+}=0$. Inserting the Klein-Gordon equation for the $B(x)$ into the formula (28), differentiated over the $x^{+}$, we have the expression

$$
\partial_{k}\left[\partial_{j} B(x)+x^{+} \partial_{j} \partial_{+} B(x)\right]+\frac{1}{2} \partial_{k}\left[x^{j}\left(\Delta_{\perp}-M^{2}\right) B(x)\right]=0,
$$

which may be integrated over the $x^{k}$, giving after some manipulations

$$
x^{+} \partial_{j} \partial_{+} B(x)+\frac{1}{2}\left(\Delta_{\perp}-M^{2}\right)\left[x^{j} B(x)\right]=0 .
$$

The first term of this pattern may be calculated from the equation (24), differentiated over the $x^{j}$ variable. It permits to write

$$
\frac{1}{2}\left(\Delta_{\perp}-M^{2}\right)\left[x^{j} B\left(x^{+}, x^{-}, \mathbf{x}_{\perp}\right)+x^{-} \partial_{j} \int_{0}^{x^{+}} d \tau B\left(\tau, x^{-}, \mathbf{x}_{\perp}\right)\right]=\frac{i}{4 \pi} \partial_{j} \delta^{(2)}\left(\mathbf{x}_{\perp}\right) .
$$

The function $B(x)$, as the scalar one, satisfies the Lorentz symmetry condition $\left(x^{\mu} \partial^{\nu}-x^{\nu} \partial^{\mu}\right) B(x)=0$. It allows to do explicit calculation of the integral in the above pattern. Therefore

$$
\left(\Delta_{\perp}-M^{2}\right)\left[x^{j} B\left(0, x^{-}, \mathbf{x}_{\perp}\right)\right]=\frac{i}{2 \pi} \partial_{j} \delta^{(2)}\left(\mathbf{x}_{\perp}\right) .
$$


Solution of this equation leads to the derivative of the modified Bessel function of the second kind

$$
x^{j} B\left(0, x^{-}, \mathbf{x}_{\perp}\right)=\frac{i}{(2 \pi)^{2}} \partial_{j} \mathrm{~K}_{0}\left(M \sqrt{\mathbf{x}_{\perp}^{2}}\right) .
$$

Including the properties of the modified Bessel functions (67), we derive the ultimate results:

$$
B(0, \bar{x})=-\frac{i}{(2 \pi)^{2}} \cdot \frac{M}{x_{\perp}} \mathrm{K}_{1}\left(M x_{\perp}\right), \quad A(0, \bar{x})=-\frac{1}{(2 \pi)^{2}} \cdot \frac{M^{2}}{x_{\perp}} \mathrm{K}_{1}\left(M x_{\perp}\right),
$$

for the contributions to the Wightman function onto the light-front hyper-surface [6-8].

Set of the Eqs. (15), (17), (18) and (19) can be readily solved in the momentum space. Therein, the mass dependent partial Fourier transforms define the Wightman function for fermions as follows

$$
\left.S_{a b}\left(x^{+}, x^{-}, \mathbf{x}_{\perp}\right)=\int \frac{d^{2} \mathbf{k}_{\perp}}{(2 \pi)^{2}} e^{-i \mathbf{k}_{\perp} \cdot \mathbf{x}_{\perp}} \int_{0}^{+\infty} \frac{d k_{-}}{2 \pi} e^{-i\left[k_{-} x^{-}+\frac{\mu^{2}\left(\mathbf{k}_{\perp}\right)}{k_{-}} x^{+}\right.}\right] \tilde{S}_{a b}\left(k_{-}, \mathbf{k}_{\perp}\right),
$$

where the mass function $\mu\left(\mathbf{k}_{\perp}\right)$ is $\mu\left(\mathbf{k}_{\perp}\right)=(1 / \sqrt{2}) \sqrt{\mathbf{k}_{\perp}^{2}+M^{2}}$. With applying the standard representation of the Dirac delta distribution, discussed set of the integro-differential equations for the Wightman function comes finally down to the algebraic form:

$$
\tilde{S}_{++}(\bar{k})=\frac{1}{\sqrt{2}} \Lambda_{+}, \quad \tilde{S}_{ \pm \mp}(\bar{k})=\frac{1}{2 \sqrt{2}} \cdot \frac{\left(\mathbf{k}_{\perp}+M\right)}{k_{-}} \gamma^{\mp}, \quad \tilde{S}_{--}(\bar{k})=\frac{1}{\sqrt{2}} \cdot \frac{\mu^{2}\left(\mathbf{k}_{\perp}\right)}{k_{-}^{2}} \Lambda_{-} .
$$

The Wightman function for scalars $\Delta_{+}(x)$ is done by the integral representation (89). From this fact one can freely reproduce the well-known correspondence $S_{ \pm \pm}(x)=i \sqrt{2} \partial_{\mp} \Delta_{+}(x) \Lambda_{ \pm}$and also $S_{ \pm \mp}(x)=$ $-(1 / \sqrt{2}) \gamma^{\mp}\left(i \not \partial_{\perp}-M\right) \Delta_{+}(x)$ between derivatives of the scalar and the fermionic Wightman functions $[9,10]$.

\section{Feynman Propagator for Fermions}

Studying the fermionic anti-commutators, we already obtained the term $\Psi_{a}(x) \Psi_{b}^{\dagger}(0)$, provided by the above Wightman functions. But the results from the CPT invariance (88) and the translational symmetry (75) enable to calculate also the second contribution $\Psi_{b}^{\dagger}(0) \Psi_{a}(x)$. For instance

$$
\left\langle 0\left|\Psi_{-}^{\dagger}(0) \Psi_{-}(x)\right| 0\right\rangle=i \sqrt{2} \partial_{+} \Delta_{+}(-x) \Lambda_{-} .
$$

Therefore, from the explicit expression of the derivative over the $x^{+}$for the scalar Wightman function (91) and from the distribution identity $1 /(x \pm \varepsilon)=\mp i \pi \delta(x)+\mathrm{P}(1 / x)$ emerges

$$
\left\langle 0\left|\left\{\Psi_{-}\left(x^{+}, \bar{x}\right), \Psi_{-}^{\dagger}\left(x^{+}, \overline{0}\right)\right\}\right| 0\right\rangle=\frac{1}{\sqrt{2}} \delta\left(x^{+}\right) \delta^{(2)}\left(\mathbf{x}_{\perp}\right) \Lambda_{-}+R\left(x^{+}, \bar{x}\right) .
$$

As it can be seen, the obtained anti-commutator is singular on the light-front hyper-surface $x^{+}=0$. It is a discrepancy between the existing light-front finite result and the outcome from the method proposed in this work. The convergent part $R(x)$ is established by the integral

$$
R\left(x^{+}, \bar{x}\right)=\frac{i}{\sqrt{2}}\left(\Delta_{\perp}-M^{2}\right) \int_{0}^{x^{-}} d \xi\left[\Delta_{+}\left(x^{+}, \xi, \mathbf{x}_{\perp}\right)-\Delta_{+}\left(-x^{+},-\xi,-\mathbf{x}_{\perp}\right)\right] \Lambda_{-},
$$

which comes from the Volterra equation for the scalar Wightman function (90). The non-divergent light-front anti-commutators (9) and (11) may be derived the same way.

Feynman propagator for the fermions is defined as the vacuum expectation value of the chronologically ordered in the time $x^{+}$bi-linear field operator. It follows

$$
S_{F a b}(x)=\left\langle 0\left|T^{+} \Psi_{a}(x) \Psi_{b}^{\dagger}(0)\right| 0\right\rangle=\theta\left(x^{+}\right) S_{a b}(x)-\theta\left(-x^{+}\right) \gamma^{5} S_{a b}(-x) \gamma^{5},
$$


according to the CPT invariance. Including representation (89) of the scalar Wightman function, the fermionic one $S_{++}(x)=i \sqrt{2} \partial_{-} \Delta_{+}(x) \Lambda_{+}$may be transformed, after the change of the variables $k_{-}=\left|x^{+}\right| \kappa, \kappa>0$ inside the integral, to the pattern

$$
S_{F++}(x)=\frac{1}{\sqrt{2}} x^{+} \int \frac{d^{2} \mathbf{k}_{\perp}}{(2 \pi)^{2}} e^{-i \mathbf{k}_{\perp} \cdot \mathbf{x}_{\perp}} \int_{0}^{+\infty} \frac{d \kappa}{2 \pi} e^{-i\left[x^{-} x^{+} \kappa+\frac{\mu^{2}\left(\mathbf{k}_{\perp}\right)}{\kappa}\right]} \Lambda_{+} .
$$

But the scalar Feynman propagator is done by the Eq. (96). It allows to put the notorious relation $S_{F++}(x)=$ $i \sqrt{2} \partial_{-} \Delta_{F}(x) \Lambda_{+}$. By the same token we have the result concerning also the $S_{F \pm \mp}(x)$ components

$$
S_{F \pm \mp}(x)=\frac{1}{2 \sqrt{2}} \int \frac{d^{2} \mathbf{k}_{\perp}}{(2 \pi)^{2}} e^{-i \mathbf{k}_{\perp} \cdot \mathbf{x}_{\perp}} \int_{0}^{+\infty} \frac{d \kappa}{2 \pi} e^{-i\left[x^{-} x^{+} \kappa+\frac{\mu^{2}\left(\mathbf{k}_{\perp}\right)}{\kappa}\right]} \frac{\left(\mathbf{k}_{\perp}+M\right)}{\kappa} \gamma^{\mp} .
$$

And considering anew the expression (96), we may compute the familiar equation, which finally yields $S_{F \pm \mp}(x)=(1 / \sqrt{2})\left(i \not \not_{\perp}+M\right) \Delta_{F}(x) \gamma^{\mp}$. But in the case of the component $S_{F--}(x)$, it is necessary to take account of, that the anti-commutator (39) remains singular onto the hyper-surface $x^{+}=0$. Therefore

$$
S_{F--}(x)=-i \sqrt{2} \delta\left(x^{+}\right)\left[\Delta_{+}(x)-\Delta_{+}(-x)\right] \Lambda_{-}+i \sqrt{2} \partial_{+} \Delta_{F}(x) \Lambda_{-}
$$

and one observes, in contrast to the convergent result from direct calculation, that the same divergence comes up at the final outcome for discussed part of the propagator for fermions [11].

\section{Renormalized Gaussian Effective Potential}

The action corresponding to the particular model may be expanded around its minimum at the point established by the certain value of the classical field. First term of this series describes the effective potential. It includes all contributions from the 1PI Feynman diagrams with the one or more loops. For this reason, the effective potential may be used for the perturbative analysis of the quantum corrections to the studied model. But the effective potential may be also handled by the Gaussian approximation, wherein it is computed as the variational minimum of the vacuum expectation value of the Hamiltonian density $V_{\text {eff }}=\langle 0|\mathcal{H}(x)| 0\rangle$ with respect to the mass of the free field. In this method one decomposes the scalar field $\phi(x)$ with the mass $m$, introducing the constant classical field $\phi_{0}=\langle 0|\phi(x)| \underset{\tilde{\phi}}{0}$ and the free field $\tilde{\phi}(x)$ with the variational mass $\mu$, according to the following relationship $\phi(x)=\phi_{0}+\tilde{\phi}(x)$. Moreover, the vacuum expectation value of the light-front Hamiltonian density for the Yukawa model with self-interacting scalar field is directly expressed by the relevant Wightman functions, without engaging the normal ordering procedure, leading finally to the non-zero result [12-14].

The canonical momenta for the Yukawa model with self-interacting scalar field: $\pi_{a}=\partial \mathcal{L} / \partial\left(\partial_{+} \psi_{a}^{\dagger}\right)$ and $\pi_{a}^{\dagger}=\partial \mathcal{L} / \partial\left(\partial_{+} \psi_{a}\right)$, where $\psi_{a}=\Psi_{a}$ or $\psi_{a}=\phi$, may be obtained from the Lorentz invariant Lagrangian density (2). This well-known method gives:

$$
\pi_{\phi}=\partial_{-} \phi, \quad \pi_{+}^{\dagger}=i \sqrt{2} \Psi_{+}^{\dagger}, \quad \pi_{-}^{\dagger}=0, \quad \pi_{+}=0, \quad \pi_{-}=0 .
$$

The Legendre transformation

$$
\mathcal{H}=\pi_{\phi}\left(\partial_{+} \phi\right)+\pi_{+}^{\dagger}\left(\partial_{+} \Psi_{+}\right)+\pi_{-}^{\dagger}\left(\partial_{+} \Psi_{-}\right)+\left(\partial_{+} \Psi_{+}^{\dagger}\right) \pi_{+}+\left(\partial_{+} \Psi_{-}^{\dagger}\right) \pi_{-}-\mathcal{L}
$$

enables to compute the light-front canonical Hamiltonian density for the studied model

$$
\begin{aligned}
\mathcal{H}= & \frac{1}{2}\left(\partial_{j} \phi\right)\left(\partial_{j} \phi\right)+\frac{1}{2} m^{2} \phi^{2}+\lambda \phi^{4}-i \sqrt{2} \Psi_{-}^{\dagger} \partial_{-} \Psi_{-}-\frac{i}{\sqrt{2}} \Psi_{-}^{\dagger} \gamma^{+} \gamma^{j} \partial_{j} \Psi_{+}-\frac{i}{\sqrt{2}} \Psi_{+}^{\dagger} \gamma^{-} \gamma^{j} \partial_{j} \Psi_{-} \\
& +\frac{1}{\sqrt{2}} M \Psi_{-}^{\dagger} \gamma^{+} \Psi_{+}+\frac{1}{\sqrt{2}} M \Psi_{+}^{\dagger} \gamma^{-} \Psi_{-}+\frac{1}{\sqrt{2}} g \phi \Psi_{-}^{\dagger} \gamma^{+} \Psi_{+}+\frac{1}{\sqrt{2}} g \phi \Psi_{+}^{\dagger} \gamma^{-} \Psi_{-} .
\end{aligned}
$$

The light-front or the equal-time calculations compel to introduce the procedure of regularization for the Fourier integral representation of the fields. It may be the cut-off in the momentum space, for example. But 
it is not a unique way to obtain the finite results. It is easy to realize, that the vacuum expectation value of Hamiltonian density for the Yukawa model, due to the translational symmetry, has the form

$$
V_{\text {eff }}=\langle 0|\mathcal{H}(x)| 0\rangle=\langle 0|\mathcal{H}(0)| 0\rangle=\frac{1}{2}\left\langle 0\left|\partial_{j} \phi(0) \partial_{j} \phi(0)\right| 0\right\rangle+\cdots .
$$

All the terms marked by the dots come from the Eq. (47). These Wightman functions contain each field fixed at the point $x=0$, what causes, that the expression (48) is divergent. One can see, the point-splitting method of regularization may be applied here. This procedure leads to certain replacement of the singular vacuum expectation value contributions. For example

$$
\left\langle 0\left|\Psi_{+}^{\dagger}(0) \Psi_{-}(0)\right| 0\right\rangle \rightarrow\left\langle 0\left|\Psi_{+}^{\dagger}(0) \Psi_{-}(x)\right| 0\right\rangle .
$$

The masses of the quantum fields in the Wightman functions are treated as the variational parameters. We introduce them as $\mu$ for the scalar and $\mathcal{M}$ for the fermionic Wightman functions $[15,16]$. Let also assume, the scalar field has the non-zero vacuum expectation value $\langle 0|\phi(x)| 0\rangle=\phi_{0}$, providing the Gaussian effective potential by the below approximated factorizations for the many-point vacuum expectation values:

$$
\langle 0|\phi(0) \phi(x)| 0\rangle=\phi_{0}^{2}+\Delta_{+}(-x ; \mu), \quad\left\langle 0\left|\phi^{2}(0) \phi^{2}(x)\right| 0\right\rangle=\phi_{0}^{4}+6 \phi_{0}^{2} \Delta_{+}(-x ; \mu)+3 \Delta_{+}^{2}(-x ; \mu) .
$$

Thus, the Gaussian effective potential for the Yukawa model in $D=1+3$ dimensions, regularized by the point-splitting procedure, satisfies

$$
\begin{aligned}
V_{\text {eff }}^{r e g}\left(\mu, \mathcal{M} ; \phi_{0}\right)= & -\frac{1}{2}\left(\Delta_{\perp}-m^{2}\right) \Delta_{+}(-x ; \mu)+\frac{1}{2} m^{2} \phi_{0}^{2}+3 \lambda \Delta_{+}^{2}(-x ; \mu)+6 \lambda \phi_{0}^{2} \Delta_{+}(-x ; \mu)+\lambda \phi_{0}^{4}+ \\
& +2\left(\Delta_{\perp}-2 M \mathcal{M}+\mathcal{M}^{2}\right) \Delta_{+}(-x ; \mathcal{M})-4 g \phi_{0} \mathcal{M} \Delta_{+}(-x ; \mathcal{M}) .
\end{aligned}
$$

From the stationary conditions for the regularized Gaussian effective potential

$$
\left.\frac{\partial}{\partial \mathcal{M}} V_{e f f}^{r e g}\left(\mu, \mathcal{M} ; \phi_{0}\right)\right|_{\mathcal{M}=\overline{\mathcal{M}}}=0=\left.\frac{\partial}{\partial \mu} V_{e f f}^{r e g}\left(\mu, \mathcal{M} ; \phi_{0}\right)\right|_{\mu=\bar{\mu}}
$$

one can find the equations for the optimum values of these variational masses $\overline{\mathcal{M}}$ and $\bar{\mu}$. In the case of the parameter $\overline{\mathcal{M}}$ it is

$$
\left\{\left[\Delta_{\perp}-\overline{\mathcal{M}}\left(2 M+2 g \phi_{0}-\overline{\mathcal{M}}\right)\right] \frac{\partial}{\partial \overline{\mathcal{M}}}-2\left[M+g \phi_{0}-\overline{\mathcal{M}}\right]\right\} \Delta_{+}(-x ; \overline{\mathcal{M}})=0 .
$$

Consistency of the regularization requires to choose the splitting parameter as the space-like four-vector $x=\left(0,0, \mathbf{x}_{\perp}\right), x^{2}<0$, where $\mathbf{x}_{\perp}^{2}>0$. The condition for $\overline{\mathcal{M}}$, due to the explicit form of the Wightman function (93) and its derivatives (94), (95), yields

$$
\left(M+g \phi_{0}-\overline{\mathcal{M}}\right)\left[\mathrm{K}_{1}\left(\overline{\mathcal{M}} x_{\perp}\right)-\left(\overline{\mathcal{M}} x_{\perp}\right) \mathrm{K}_{0}\left(\overline{\mathcal{M}} x_{\perp}\right)\right]=0 .
$$

The regularization independent solution of this equation satisfies

$$
\overline{\mathcal{M}}=M+g \phi_{0}
$$

In the same way, for the mass $\bar{\mu}$ we have

$$
\left[\left(\Delta_{\perp}-m^{2}-12 \lambda \phi_{0}^{2}\right)-12 \lambda \Delta_{+}(-x ; \bar{\mu})\right] \frac{\partial}{\partial \bar{\mu}} \Delta_{+}(-x ; \bar{\mu})=0 .
$$

After the insertion of the explicit expressions for the Wightman function (93), (94) and (95) to the above formula, it transforms to

$$
\left(\bar{\mu} x_{\perp}\right) \mathrm{K}_{0}\left(\bar{\mu} x_{\perp}\right)\left[\left(\bar{\mu} x_{\perp}\right) \mathrm{K}_{1}\left(\bar{\mu} x_{\perp}\right)+\frac{\pi^{2}}{3 \lambda}\left(m^{2}+12 \lambda \phi_{0}^{2}-\bar{\mu}^{2}\right) x_{\perp}^{2}\right]=0 .
$$


The trivial root $\bar{\mu}=0$ emerges from the first factorized part $\left(\bar{\mu} x_{\perp}\right) \mathrm{K}_{0}\left(\bar{\mu} x_{\perp}\right)=0$, whereas the function $\mathrm{K}_{0}\left(\bar{\mu} x_{\perp}\right)$ is positive. It is easy to rewrite (57) as the patterns:

$$
\alpha z \mathrm{~K}_{1}(z)-z^{2}+\beta x_{\perp}^{2}=0, \quad z=\bar{\mu} x_{\perp}, \quad \alpha=\frac{3 \lambda}{\pi^{2}}, \quad \beta=m^{2}+12 \lambda \phi_{0}^{2} .
$$

The conditions of our effective potential renormalization call for the bare scalar field coupling constant yielding $\lambda=0$. Therefore, it is necessary to solve the Eq. (58) in the limit $\lambda \rightarrow 0$. At the end, we derive the regularization independent solution

$$
\bar{\mu}=m .
$$

By substitution of the optimum values for the variational parameters (55) and (59) to the Gaussian effective potential for the Yukawa model, we will have in its minimum

$$
\begin{aligned}
V_{\text {eff }}^{\text {reg }}\left(m, \overline{\mathcal{M}} ; \phi_{0}\right)= & -\frac{1}{2}\left(\Delta_{\perp}-m^{2}\right) \Delta_{+}(-x ; m)+\frac{1}{2} m^{2} \phi_{0}^{2} \\
& +3 \lambda \Delta_{+}^{2}(-x ; m)+6 \lambda \phi_{0}^{2} \Delta_{+}(-x ; m)+\lambda \phi_{0}^{4}+2\left(\Delta_{\perp}-\overline{\mathcal{M}}^{2}\right) \Delta_{+}(-x ; \mathcal{M}) .
\end{aligned}
$$

According to (93) and (94), the above bare potential with the point-splitting regularization parameter $x=$ $\left(0,0, \mathbf{x}_{\perp}\right)$ can be showed with help of the modified Bessel functions

$$
\begin{aligned}
V_{\text {eff }}^{\text {reg }}\left(m_{B}, \overline{\mathcal{M}}_{B} ; \phi_{0}\right)= & -\frac{1}{(2 \pi)^{2}} \cdot \frac{m_{B}^{2}}{x_{\perp}^{2}} \mathrm{~K}_{2}\left(m_{B} x_{\perp}\right)+\frac{1}{2} m_{B}^{2} \phi_{0}^{2} \\
& +\frac{3}{(2 \pi)^{4}} \lambda \frac{m_{B}^{2}}{x_{\perp}^{2}} \mathrm{~K}_{1}^{2}\left(m_{B} x_{\perp}\right)+\frac{6}{(2 \pi)^{2}} \lambda \phi_{0}^{2} \frac{m_{B}}{x_{\perp}} \mathrm{K}_{1}\left(m_{B} x_{\perp}\right) \\
& +\lambda_{B} \phi_{0}^{4}+\frac{1}{\pi^{2}} \cdot \frac{\overline{\mathcal{M}}_{B}^{2}}{x_{\perp}^{2}} \mathrm{~K}_{2}\left(\overline{\mathcal{M}}_{B} x_{\perp}\right) .
\end{aligned}
$$

The renormalization scheme in this case leads to the same physical masses as the bare parameters $M=M_{B}$ and $m=m_{B}$. The consistency of the renormalization prescription requires also, that the bare coupling constants are $g_{B}=0$ and $\lambda_{B}=0$. We are proceeding with the extra linear term $a_{B} \phi_{0}$ added to the potential $V \rightarrow V+a_{B} \phi_{0}$. Thus, the renormalization condition satisfies

$$
a_{R}=\left.\frac{\partial}{\partial \phi_{0}} V_{e f f}^{r e g}\left(m_{B}, \overline{\mathcal{M}}_{B}, g_{B}, \lambda_{B} ; \phi_{0}\right)\right|_{\phi_{0}=0}=0 .
$$

It permits to hold on the renormalized effective potential without the term linear in $\phi_{0}$. Employing the expansions of the Bessel functions (68) and (69), we obtain

$$
\begin{aligned}
V_{e f f}^{r e g}\left(m, M, g_{B}, \lambda_{B} ; \phi_{0}\right)= & V_{\infty}-\frac{c_{3}}{8(2 \pi)^{2}} m^{4}+\frac{1}{2} m^{2} \phi_{0}^{2}+\lambda_{B} \phi_{0}^{4}+a_{B} \phi_{0} \\
& +\frac{3 \lambda_{B}}{(2 \pi)^{4}} \cdot \frac{m^{2}}{x_{\perp}^{2}}\left\{\frac{1}{m^{2} x_{\perp}^{2}}+\left[-\ln \left(\frac{m x_{\perp}}{2}\right)+c_{0}\right]\right. \\
& \left.+\frac{1}{32} m^{2} x_{\perp}^{2}\left[8 \ln ^{2}\left(m x_{\perp}\right)+c_{1} \ln \left(m x_{\perp}\right)+c_{2}\right]\right\} \\
& +\frac{6 \lambda_{B} \phi_{0}^{2}}{(2 \pi)^{2}} \cdot \frac{m}{x_{\perp}}\left\{\frac{1}{m x_{\perp}}+\frac{m x_{\perp}}{2}\left[-\ln \left(\frac{m x_{\perp}}{2}\right)+c_{0}\right]\right\} \\
& +\frac{c_{3}}{2(2 \pi)^{2}}\left(M+g_{B} \phi_{0}\right)^{4}-\frac{\left(M+g_{B} \phi_{0}\right)^{2}}{2 \pi^{2} x_{\perp}^{2}} .
\end{aligned}
$$

The term containing $g_{B}^{2}$ in this expression is divergent as $1 / x_{\perp}^{2}$. In turn, one has the singularity behaving as $1 / x_{\perp}^{4}$ for the contribution with $\lambda_{B}$. Hence, we renormalize the coupling constants $g$ and $\lambda$ by the introduction: 


$$
G=g_{R}=\frac{g_{B}}{x_{\perp}}, \quad \Lambda^{4}=\lambda_{R}=\frac{\lambda_{B}}{x_{\perp}^{4}}
$$

Thus, the parameter $a_{B}$, defined by (62), may be written as $a_{B}=\left(g_{B} M\right) /\left(\pi^{2} x_{\perp}^{2}\right)$. It makes possible cancellation of the term $\left(g_{B} \phi_{0} M\right) /\left(\pi^{2} x_{\perp}^{2}\right)$ in the discussed potential. Finally, in the limit $x_{\perp} \rightarrow 0$, we have the regularized Gaussian potential for the Yukawa model in $D=1+3$ dimensions, yielding

$$
V_{e f f}^{r e g}\left(m, M, G, \Lambda ; \phi_{0}\right)=V_{\infty}+\frac{1}{2}\left(m^{2}-2 \frac{G^{2}}{\pi^{2}}\right) \phi_{0}^{2}-\frac{c_{3}}{8(2 \pi)^{2}} m^{4}+\frac{c_{3}}{2(2 \pi)^{2}} M^{4}+\frac{3}{(2 \pi)^{4}} \Lambda^{4} .
$$

The term $V_{\infty}$ describes constant infinite density of the vacuum energy in this model.

\section{Conclusions}

The light-front Yukawa model with the extra term of the self-interacting scalar field in $D=1+3$ dimensions is the extension and generalization of the $D=1+1$ case. For both, there are no classical analogues. The model in $D=1+3$ dimensions was studied with the help of such the way of quantization, where the algebra of the (anti) commutators was inferred from the Heisenberg equations, induced by the kinematical generating operator of the translation $P^{+}$. In this approach the Heisenberg equations were the point of departure for obtaining mentioned algebra of the (anti-) commutators. It unveiled some discrepancies between existing and proposed method of the quantization. The light-front Wightman function for fermions was derived from the integro(partial) differential equations, accommodating the Lorentz and the CPT symmetry. The role of the Lorentz and the CPT symmetries was here especially emphasized. Discussed method did not require introduction either the Fock states or the Fourier representation of the field operators for derivation the light-front Wightman function for the free fermions. The propagator for the fermions was obtained from the Wightman function, providing the CPT invariance. The renormalized Hamiltonian of this model was calculated by the space-like point-splitting regularization of the stationary Gaussian effective potential, which was directly expressed by the relevant Wightman functions, without engaging the normal ordering procedure. This approach was finally lead to the non-zero result. The method presented here could be tested on the light-front solvable and other models.

Open Access This article is distributed under the terms of the Creative Commons Attribution 4.0 International License (http:// creativecommons.org/licenses/by/4.0/), which permits unrestricted use, distribution, and reproduction in any medium, provided you give appropriate credit to the original author(s) and the source, provide a link to the Creative Commons license, and indicate if changes were made.

\section{Appendix A: Light-Front Coordinates}

In $D=1+3$ dimensions, we use the light-front coordinates: $x=\left(x^{+}, x^{-}, \mathbf{x}_{\perp}\right), x^{ \pm}=(1 / \sqrt{2})\left(x^{0} \pm x^{3}\right), \mathbf{x}_{\perp}=$ $\left(x^{1}, x^{2}\right)$, supplemented by the following abbreviations $\bar{x}=\left(x^{+}=0, x^{-}, \mathbf{x}_{\perp}\right)$ and $x=\left(x^{+}, x^{-}=0, \mathbf{x}_{\perp}\right)$. Analogous definitions are valid for the gamma matrices. Remarkably, we have $\left(\gamma^{ \pm}\right)^{\frac{1}{2}}=0$ and $\left(\gamma^{ \pm}\right)^{\dagger}=\gamma^{\mp}$. The components of the metric tensor for the light-front coordinates are $g_{+-}=1=g_{-+}$and moreover $g_{++}=0=g_{--}$, where $g_{j k}=-\delta_{j k}$ for $j, k=1,2$. They lead to the up and down index rules $a_{ \pm}=a^{\mp}$ and also $a_{j}=-a^{j}$. The scalar product of the four-vectors satisfies $a \cdot b=a_{+} b^{-}+a_{-} b^{+}-\mathbf{a}_{\perp} \cdot \mathbf{b}_{\perp}$ with $\mathbf{a}_{\perp} \cdot \mathbf{b}_{\perp}=a_{j} b_{j}$. We also apply the shortcut for the following sum $\boldsymbol{q}_{\perp}=a_{j} \gamma^{j}$. The light-front derivatives $\partial_{ \pm}=\partial / \partial x_{ \pm}$arrange the Dalembertian operator $\square=2 \partial_{+} \partial_{-}-\Delta_{\perp}$, where is $\Delta_{\perp}=\partial_{j} \partial_{j}$. The field operator decomposition onto the + and - components is performed in the base including constant bispinors $u_{ \pm}$. Their tensor products $u_{ \pm} u_{ \pm}^{\dagger}=\Lambda_{ \pm}=u_{ \pm}^{\dagger} u_{ \pm}$give the operators $\Lambda_{ \pm}$, which define the $\Psi_{ \pm}$fields $\Psi_{ \pm}=\Lambda_{ \pm} \Psi$ and $\Psi=\Psi_{+}+\Psi_{-}$. These $\Lambda_{ \pm}$operators are projective and hermitian. They may be also expressed by the $\gamma^{ \pm}$ matrices: $\Lambda_{+}+\Lambda_{-}=\mathrm{I}, \Lambda_{ \pm}^{2}=\Lambda_{ \pm}, \Lambda_{ \pm} \Lambda_{\mp}=0, \Lambda_{ \pm}^{\dagger}=\Lambda_{ \pm}, \Lambda_{ \pm}=(1 / 2) \gamma^{\mp} \gamma^{ \pm}$. These $\Lambda_{ \pm}$operators commute with the $\gamma^{j}$ matrices and satisfy the two following formulae $\gamma^{ \pm} \Lambda_{ \pm}=\gamma^{ \pm}=\Lambda_{\mp} \gamma^{ \pm}$. It is due to the fact, that the matrices $\gamma^{ \pm}$may be introduced on the same way as the $\Lambda_{ \pm}$operators $u_{\mp} u_{ \pm}^{\dagger}=\gamma^{ \pm}=u_{ \pm}^{\dagger} u_{\mp}$. 
Therefore, the $\gamma^{ \pm}$play the analogous role as the $\Lambda_{ \pm}$for the anti-commutators of $\Psi$ fields with the different + and - indexes. The commutator of the gamma matrices $s_{\mu \nu}=(i / 4)\left[\gamma_{\mu}, \gamma_{\nu}\right]$ defines the operator of the spin for the fermionic field.

\section{Appendix B: Selected Properties of Modified Bessel Functions of the Second Kind}

The modified Bessel functions of the second kind $\mathrm{K}_{n}(z)=\mathrm{K}_{-n}(z)$ are the solutions of the differential equation

$$
\left(\frac{d^{2}}{d z^{2}}+\frac{1}{z} \frac{d}{d z}-1-\frac{n^{2}}{z^{2}}\right) \mathrm{K}_{n}(z)=0 .
$$

The useful relationships for these functions embrace:

$$
\frac{d}{d z} \mathrm{~K}_{n}(z)=-\frac{1}{2}\left[\mathrm{~K}_{n-1}(z)+\mathrm{K}_{n+1}(z)\right], \quad \mathrm{K}_{n+1}(z)=\mathrm{K}_{n-1}(z)+\frac{2 n}{z} \mathrm{~K}_{n}(z) .
$$

Expansion of the modified Bessel functions of the second kind for $|z| \ll 1$ gives:

$$
\begin{aligned}
& \mathrm{K}_{1}(z) \simeq \frac{1}{z}+\frac{1}{2} z\left[-\ln \left(\frac{z}{2}\right)+c_{0}\right], \quad \mathrm{K}_{2}(z) \simeq \frac{2}{z^{2}}-\frac{1}{2}+\frac{1}{8} z^{2}\left[-\ln (z)+c_{3}\right], \\
& \mathrm{K}_{1}^{2}(z) \simeq \frac{1}{z^{2}}+\left[-\ln \left(\frac{z}{2}\right)+c_{0}\right]+\frac{1}{32} z^{2}\left[8 \ln ^{2}(z)+c_{1} \ln (z)+c_{2}\right],
\end{aligned}
$$

where:

$$
\begin{aligned}
& c_{0}=-\frac{1}{2}+\gamma_{E}, \quad c_{1}=-4\left[1+4 \ln (2)-4 \gamma_{E}\right], \\
& c_{2}=-3+4\left[1+2 \ln (2)-4 \gamma_{E}\right] \ln (2)+4 \gamma_{E}\left(-1+2 \gamma_{E}\right), \quad c_{3}=\frac{3}{4}+\ln (2)-\gamma_{E}
\end{aligned}
$$

and $\gamma_{E}$ is the Euler constant.

\section{Appendix C: Symmetries of Wightman Function for Fermions}

In our model we put on being just one state of the Lorentz, the CPT and the translational invariant vacuum, which eigenvalues of the total energy and momenta in all directions are equal to zero:

$$
U(d, \omega, L)|0\rangle=|0\rangle, \quad \theta|0\rangle=|0\rangle, \quad P^{\mp}|0\rangle=0, \quad P^{j}|0\rangle=0 .
$$

Herein, the $U(d, \omega, L)$ is the element from the Poincaré group and $\theta$ denotes the operator of the CPT symmetry. The two-point Wightman function for fermions, which is the vacuum expectation value of the bi-linear combination for the field operators, obey:

$$
S(x, y)=\left\langle 0\left|\Psi(x) \Psi^{\dagger}(y)\right| 0\right\rangle, \quad S_{a b}(x, y)=\left\langle 0\left|\Psi_{a}(x) \Psi_{b}^{\dagger}(y)\right| 0\right\rangle, \quad a, b=-,+.
$$

Considering the shift of the field operator in the space-time

$$
\Psi(x)=e^{i P \cdot x} \Psi(0) e^{-i P \cdot x}
$$

and the translational invariance of the vacuum (72), one has

$$
\left\langle 0\left|\Psi(x) \Psi^{\dagger}(y)\right| 0\right\rangle=\left\langle 0\left|\Psi(x-y) \Psi^{\dagger}(0)\right| 0\right\rangle=\left\langle 0\left|\Psi(0) \Psi^{\dagger}(y-x)\right| 0\right\rangle .
$$

It means, that $S(x, y)=S(x-y)$. Moreover, the relationship

$$
\left\langle 0\left|\Psi(x) \Psi^{\dagger}(y)\right| 0\right\rangle=\left\langle 0\left|\Psi(0) e^{-i P \cdot(x-y)} \Psi^{\dagger}(0)\right| 0\right\rangle
$$


guarantees the analyticity of the discussed Wightman functions $\left(x^{\mu}-y^{\mu} \rightarrow x^{\mu}-y^{\mu}-i \varepsilon\right)$ under the condition, that all generators $P$ have the positive spectra. By the same reasons it is easy to prove, taking account of the Heisenberg equations, that the derivatives of the Wightman function for the fermions satisfy

$$
\left\langle 0\left|\partial_{\mu}^{x} \Psi(x) \Psi^{\dagger}(y)\right| 0\right\rangle=-\left\langle 0\left|\Psi(x) \partial_{\mu}^{y} \Psi^{\dagger}(y)\right| 0\right\rangle,
$$

for any light-front indexes $\mu=-,+, j$, where $j=1,2$.

The element from the homogenous $(d=0)$ Poincaré group $U(\omega, L)$ acts on the field operator of the fermions, giving the transformed bispinor:

$$
\Psi^{\prime}\left(x^{\prime}\right)=U^{-1}(\omega, L) \Psi(x) U(\omega, L), \quad x^{\prime}=L x, \quad \Psi^{\prime}\left(x^{\prime}\right)=R(\omega, L) \Psi(x) .
$$

Herein, $L$ represents the Lorentz matrix transforming the four-coordinates. The matrices $L$ and $R$ in the infinitesimal case $\omega^{\mu \nu} \rightarrow \delta \omega^{\mu \nu}$ follow:

$$
L_{\nu}^{\mu}=\delta_{\nu}^{\mu}-\delta \omega_{\nu}^{\mu}, \quad R(\delta \omega, L)=1+\frac{1}{2} \delta \omega^{\mu \nu}\left(x_{\mu} \partial_{\nu}-x_{\nu} \partial_{\mu}\right)-\frac{i}{2} \delta \omega^{\mu \nu} s_{\mu \nu},
$$

where $s_{\mu \nu}$ denotes the spin operator for the fermionic field. For any $\delta \omega^{\mu \nu}$ we obtain the Lorentz symmetry condition for the Wightman function

$$
\left(x_{\mu} \partial_{\nu}-x_{\nu} \partial_{\mu}\right) S(x)-i s_{\mu \nu} S(x)+i S(x) s_{\mu \nu}^{\dagger}=0 .
$$

This formula enables to write specific equations for given Lorentz and projective indexes. Below, there are collected conditions for the Wightman function of the fermions, emerging from the Lorentz symmetry requirement (80). For the abbreviation, we introduced the differential operator $\mathcal{D}^{\mu \nu}(x)=\left(x^{\mu} \partial^{\nu}-x^{\nu} \partial^{\mu}\right)$. By putting $\mu, v=+,-$ in the Eq. (80), one has:

$$
\mathcal{D}^{+-}(x) S_{ \pm \pm}(x) \mp S_{ \pm \pm}(x)=0, \quad \mathcal{D}^{+-}(x) S_{ \pm \mp}(x)=0 .
$$

For $\mu, v=+, j$, the formulae satisfy:

$$
\mathcal{D}^{+j}(x) S_{++}(x)+\frac{1}{2} \gamma^{+} \gamma^{j} S_{++}(x)+\frac{1}{2} S_{++}(x) \gamma^{-} \gamma^{j}=0, \quad \mathcal{D}^{+j}(x) S_{--}(x)=0
$$

and also:

$$
\mathcal{D}^{+j}(x) S_{+-}(x)+\frac{1}{2} \gamma^{+} \gamma^{j} S_{+-}(x)=0, \quad \mathcal{D}^{+j}(x) S_{-+}(x)+\frac{1}{2} S_{-+}(x) \gamma^{-} \gamma^{j}=0 .
$$

In the case of $\mu, v=-, j$, the Lorentz symmetry equation gives:

$$
\mathcal{D}^{-j}(x) S_{++}(x)=0, \quad \mathcal{D}^{-j}(x) S_{--}(x)+\frac{1}{2} \gamma^{-} \gamma^{j} S_{--}(x)+\frac{1}{2} S_{--}(x) \gamma^{+} \gamma^{j}=0
$$

and the two remaining conditions for the Wightman function contributions:

$$
\mathcal{D}^{-j}(x) S_{+-}(x)+\frac{1}{2} S_{+-}(x) \gamma^{+} \gamma^{j}=0, \quad \mathcal{D}^{-j}(x) S_{-+}(x)+\frac{1}{2} \gamma^{-} \gamma^{j} S_{-+}(x)=0 .
$$

By choosing two different indexes $\mu, v=j, k$, one obtains:

$$
\mathcal{D}^{j k}(x) S_{a b}(x)+\frac{1}{2}\left[\gamma^{j} \gamma^{k}, S_{a b}(x)\right]=0, \quad a, b=-,+.
$$

It exhausts the complete set of the Lorentz symmetry conditions for the Wightman function of the fermions. The anti-linear and the anti-unitary CPT operator acts on the bispinor field and leads to

$$
\theta \Psi(x) \theta^{\dagger}=i \gamma^{5} \Psi^{\dagger}(-x)
$$

Due to the vacuum CPT symmetry, the Wightman function for the fermions must satisfy the relation

$$
\left\langle 0\left|\Phi_{a}(x) \Psi_{b}^{\dagger}(y)\right| 0\right\rangle=\gamma^{5}\left\langle 0\left|\Psi_{b}^{\dagger}(-y) \Phi_{a}(-x)\right| 0\right\rangle \gamma^{5},
$$

which is valid for the different or the same bispinor fields $\Phi$ and $\Psi$. 


\section{Appendix D: Scalar Wightman Function and Propagator}

The integral representation of the Wightman function for the scalars follows

$$
\Delta_{+}(x)=\frac{1}{2} \int \frac{d^{2} \mathbf{k}_{\perp}}{(2 \pi)^{2}} e^{-i \mathbf{k}_{\perp} \cdot \mathbf{x}_{\perp}} \int_{0}^{+\infty} \frac{d k_{-}}{2 \pi} e^{-i\left[k_{-} x^{-}+\frac{\mu^{2}\left(\mathbf{k}_{\perp}\right)}{k_{-}} x^{+}\right]} \frac{1}{k_{-}} .
$$

The Wightman function for the scalars in the light-front coordinates satisfies the Volterra integral equation

$$
\begin{aligned}
& \Delta_{+}\left(x^{+}, x^{-}, \mathbf{x}_{\perp}\right) \\
& =-\frac{1}{2 \pi} \gamma_{E} \delta^{(2)}\left(\mathbf{x}_{\perp}\right)-\frac{i}{8}\left[\operatorname{sgn}\left(x^{+}\right)+\operatorname{sgn}\left(x^{-}\right)\right] \delta^{(2)}\left(\mathbf{x}_{\perp}\right)-\frac{1}{4 \pi} \ln \left(\frac{m^{2}\left|x^{+} x^{-}\right|}{2}\right) \delta^{(2)}\left(\mathbf{x}_{\perp}\right) \\
& -\frac{1}{(2 \pi)^{2}} \partial_{j}\left[\frac{x_{j}}{x_{\perp}^{2}} \mathrm{~K}_{0}\left(m x_{\perp}\right)\right]+\frac{1}{2}\left(\Delta_{\perp}-m^{2}\right) \int_{0}^{x^{+}} d \tau \int_{0}^{x^{-}} d \xi \Delta_{+}\left(\tau, \xi, \mathbf{x}_{\perp}\right), \quad x \neq 0 .
\end{aligned}
$$

The derivative over the $x^{+}$variable of the light-front Wightman function for the scalars obeys the integro(partial) differential equation

$$
\partial_{+} \Delta_{+}\left(x^{+}, x^{-}, \mathbf{x}_{\perp}\right)=-\frac{1}{4 \pi} \cdot \frac{1}{x^{+}-\varepsilon} \delta^{(2)}\left(\mathbf{x}_{\perp}\right)+\frac{1}{2}\left(\Delta_{\perp}-m^{2}\right) \int_{0}^{x^{-}} d \xi \Delta_{+}\left(x^{+}, \xi, \mathbf{x}_{\perp}\right)
$$

For the derivative over the $x^{-}$, one has the analogous pattern

$$
\partial_{-} \Delta_{+}\left(x^{+}, x^{-}, \mathbf{x}_{\perp}\right)=-\frac{1}{4 \pi} \cdot \frac{1}{x^{-}-\varepsilon} \delta^{(2)}\left(\mathbf{x}_{\perp}\right)+\frac{1}{2}\left(\Delta_{\perp}-m^{2}\right) \int_{0}^{x^{+}} d \tau \Delta_{+}\left(\tau, x^{-}, \mathbf{x}_{\perp}\right) .
$$

The scalar Wightman function for the space-like $x^{2}<0$ four-vector $x=\left(0,0, \mathbf{x}_{\perp}\right)$, where $x_{\perp}^{2}=\mathbf{x}_{\perp}^{2}>0$, comes down to

$$
\Delta_{+}\left(0,0, \mathbf{x}_{\perp} ; m\right)=\Delta_{+}\left(\mathbf{x}_{\perp} ; m\right)=\frac{1}{(2 \pi)^{2}} \cdot \frac{m}{x_{\perp}} \mathrm{K}_{1}\left(m x_{\perp}\right) .
$$

Thus, the Eq. (66) allows to derive

$$
\left(\Delta_{\perp}-m^{2}\right) \Delta_{+}\left(\mathbf{x}_{\perp} ; m\right)=\frac{2}{(2 \pi)^{2}} \cdot \frac{m^{2}}{x_{\perp}^{2}} \mathrm{~K}_{2}\left(m x_{\perp}\right), \quad x_{\perp}>0 .
$$

Due to the relationships (67), the mass derivatives of the Wightman function yield:

$$
\frac{\partial}{\partial m} \Delta_{+}\left(\mathbf{x}_{\perp} ; m\right)=-\frac{m}{(2 \pi)^{2}} K_{0}\left(m x_{\perp}\right), \quad\left(\Delta_{\perp}-m^{2}\right) \frac{\partial}{\partial m} \Delta_{+}(\mathbf{x} ; m)=0, \quad x_{\perp}>0 .
$$

The Feynman propagator of the scalar field is described by the formula

$$
\Delta_{F}(x)=\frac{1}{2} \int \frac{d^{2} \mathbf{k}_{\perp}}{(2 \pi)^{2}} e^{-i \mathbf{k}_{\perp} \cdot \mathbf{x}_{\perp}} \int_{0}^{+\infty} \frac{d \kappa}{2 \pi} e^{-i\left[x^{-} x^{+} \kappa+\frac{\mu^{2}\left(\mathbf{k}_{\perp}\right)}{\kappa}\right]} \frac{1}{\kappa},
$$

for the argument $x \neq 0$. 


\section{References}

1. P.A.M. Dirac, Generalized Hamiltonian dynamics. Can. J. Phys. 2, 129-148 (1950)

2. P.A.M. Dirac, Lectures on Quantum Mechanics (Yeshiva University Press, New York, 1964)

3. T. Heinzl, Lecture Notes in Physics, vol. 572 (Springer, Berlin, 2001), p. 55

4. M. Burkardt, Light front quantization. Adv. Nucl. Phys. 23, 1-74 (1996)

5. S.J. Brodsky, H.-C. Pauli, S. Pinsky, Quantum chromodynamics and other field theories on the light cone. Phys. Lett. C Phys. Rep. 301, 299-486 (1998)

6. J.A. Przeszowski, Lorentz symmetry for the light-front Wightman functions. Acta Phys. Pol. Proc. Suppl. B 6, 327-333 (2013)

7. J.A. Przeszowski, J. Żochowski, Scale and Lorentz transformations at the light front. Few Body Syst. 55, 485-491 (2014)

8. J.A. Przeszowski, J. Żochowski, Light-front quantization with explicit Lorentz symmetry for Yukawa model. Few Body Syst. 56, 579-585 (2015)

9. L. Martinovic, M. Luban, Analytic solution of the microcausality problem in discretized light-cone quantization. Phys. Lett. B 605, 203-213 (2005)

10. N. Nakanishi, H. Yabuki, Null-plane quantization and Haag's theorem. Lett. Math. Phys. 1, 371-374 (1977)

11. J.A. Przeszowski, E. Dzimida-Chmielewska, J. Żochowski, Light-front perturbations without spurious singularities. Few Body Syst. 57, 527-532 (2016)

12. P.J. Steinhardt, Problems of quantization in the infinite momentum frame. Ann. Phys. 128, $425-447$ (1980)

13. A. Harindranath, J.P. Vary, Variational calculation of the spectrum of two-dimensional $\phi^{4}$ theory in light-front field theory. Phys. Rev. D 37, 3010-3013 (1988)

14. E.A. Bartnik, S. Głazek, Light-front variational approach to scalar field theories. Phys. Rev. D 39, 1249-1250 (1989)

15. P.M. Stevenson, G.A. Hajj, J.F. Reed, Fermions and the Gaussian effective potential. Phys. Rev. D 34, 3117-3127 (1986)

16. R. Koniuk, R. Tarrach, Scalar field theory in 3+1 dimensions. Phys. Rev. D 31, 3178-3182 (1985) 